\title{
Twelfth Night ou les noces de la nuit et de la fête : du théâtre à l'écran
}

\section{Raphaëlle Costa de Beauregard}

\section{(2) OpenEdition}

\section{Journals}

\section{Édition électronique}

URL : http://journals.openedition.org/shakespeare/195

DOI : 10.4000/shakespeare.195

ISSN : 2271-6424

Éditeur

Société Française Shakespeare

\section{Édition imprimée}

Date de publication : 1 novembre 1998

Pagination : 37-55

ISBN : 2-84269-230-6

\section{Référence électronique}

Raphaëlle Costa de Beauregard, «Twelfth Night ou les noces de la nuit et de la fête : du théâtre à

l'écran », Actes des congrès de la Société française Shakespeare [En ligne], 16 | 1998, mis en ligne le 01 novembre 2007, consulté le 27 avril 2019. URL : http://journals.openedition.org/shakespeare/195 ; DOI : 10.4000/shakespeare.195 


\section{S H A K E S P E A R E \\ \& $\quad$ L E $\quad$ C I N É M A}

Société Française Shakespeare

Actes du Congrès de 1998

米 $*$ *

Textes réunis et présentés par

Patricia DORVAL

publiés sous la direction de

Jean-Marie MAGUIN 
Site web : <http : //alor.univ-montp3.fr/serinf/SFS/> Liste de diffusion : <sfs-1@smrl.univ-montp3.fr >

Tous droits de traduction, de reproduction et d'adaptation réservés pour tous les pays.

(C) 1998. Société Française Shakespeare,

École Normale Supérieure, 45 rue d'Ulm. 75005 Paris.

ISBN 2-84269-230-6 


\section{TW ELLFTH N I GH T \\ OU LES NOCES DE LA NUIT ET DE LA F E TE : D U T HÉ ÂTRE À L'ÉCRA N}

La comparaison entre trois versions filmées de Twelfth Night ${ }^{\prime}$ montre la récurrence d'un certain nombre de problèmes de transposition de la pièce à l'écran que nous examinerons du point de vue de la réception du spectatcur, celui du metteur en scène restant ouvert à toutes les innovations. L'espace cl le temps ainsi que le corps de l'acteur sont les principaux modes d'expression du théâtre et du cinéma; le concept même de théâtre filmć pose le problème de l'hybridation des genres, alors que celui de texte spectaculaire oriente la critique vers une lecture diversifiée de chaque ouvre per se. Quant à la sempiternelle querelle entre les adeptes de l'adaptation fidèle et les champions de la transposition interprétative, elle trouvera là un terrain fertile en rebondissements.

\section{Le théâtre filmé}

Nous commencerons par quelques remarques sur l'espace de la représentation, et le théâtre filmé, dans les trois versions étudiées. La pièce elle-même pose d'emblée les enjeux de l'illusion merveilleuse :

Viola. What country, friends, is this ?

Captain. This is Illyria, lady.

Viola. And what should I do in Illyria?

My brother he is in Elysium. 
La question de la représentation de cette Illyrie apparue comme en un songe à Viola, à la fois espace où vivent des personnages et lieu spectaculaire où toutes les métamorphoses sont possibles, est bien entendu centrale aux trois films, et permettra d'examiner ici quelques unes des contraintes inherrentes au théâtre comme au cinéma.

La représentation théâtrale se déroule dans un espace à trois dimensions régi par les lois du corps humain se déplaçant dans cet espace. Le cinéma, lui, cst dćfini par une double représentation : une représentation dite «profilmique" " où évoluent les acteurs dans un décor de studio (ou un décor naturel envisagé comme site du tournage) : c'est la mise en scène (d'où la possibilité d'un genre hybride: le théâtre filmé) et un écran à deux dimensions régi par les lois de l'image.

Unc étude comparée des trois films - 1981, 1988 et 1997 - montre que le décor de studio (le réel pro-filmique) peut soit effacer la source théâtrale du spectacle filmé, soit au contraire l'afficher pour en tirer une mise en abyme de la théâtralité même de ce spectacle, ce qui sape l'effet de réel traditionnellement considéré comme l'un des ingrédients de l'effet cinéma ${ }^{3}$.

Il importe de rappeler que les versions de 1981 et 1988 ont été tournées pour la télévision et celle de 1997 pour le grand écran: celle de 1981 souližne la complicité entre les chambres filmées et la chambre ou le salon où se trouve le spectateur, celle de 1988 nie cette complicité avec force.

\section{Le décor}

Le décor de la version de 1981 joue un rôle important dans la naturalisation de l'invraisemblable. Le générique annonce le nom des vedettes sur fond de toiles peintes inspirées d'enluminures bourguignonnes (le spectatcur du petit écran est entouré de livres ?). Elles se superposent l'une à l'autre à la manière d'un livre d'image sur fond de musique de chambre haroque (la Renaissance pour le public de 1981 ?).

Or, lorsque Viola entre dans le champ à droite, elle donne l'impression d'émerger du décor peint. Elle passe entre la toile du décor et un arbre au premier plan puis va s'asseoir sur les marches : ce qui paraissait entièrement bi-dimensionncl à première vue - toile de fond sur laquelle apparaissait le nom des acteurs et l'arbre, les marches, et même le sol - devient brusquement décor reconstitué en trois dimensions. Par ailleurs, le flou sur l'arrière plan lorsque Viola s'approche de la caméra connote la profondeur de champ, cc qui a pour effet d'indiquer également la transformation d'un espace cinématographique à deux dimensions (la pellicule photosensible du film) en espace en trois dimensions (l'espace filmique de la diégèse).

Dès la scène suivante, nous entrons dans un décor qui, cette fois-ci, affiche sa qualité de décor construit. Les acteurs font le tour de «vraics/fausses» colonnes, Olivia apparaît à la fenêtre d'une façade de décor et 
Orsino exprime son émotion sous une véritable arcade. Cependant, le cadrage serré élimine ce qui aurait trahi les frontières du plateau de tournage. L'impression créée est celle d'une place de village en miniature, sans ciel ni profondeur, le son mat et étouffé des voix aggravant encore la claustrophobic du spectateur. L'espace de la représentation est donc ici affiché, mais naturalise comme «théâtrale» la scène représentée. La fiction (de l'histoire) tire sa cohérence de la fiction même (de la représentation au théâtre).

La version de 1988 prend également le parti de souligner le décor de théâtre. Toutes les entrées et les sorties des personnages sont limitées à une seule et unique porte : celle-ci change de valeur au fil des scènes, devenant tantôt porte d'entrée, tantôt porte d'intérieur, de sorte que la terrasse où se déroule toute la pièce transpose en extérieurs de sucre glacé les chaudes intimités de la version de 1981. La polysémie du décor et la variation de ses signifiés selon son utilisation - trait caractéristique de tout décor de théâtre - est soulignée pendant tout le film.

On pourrait penser qu'il s'agit ici autant de théâtre filmé que dans la version de 1981. Et pourtant cette dernière dispose de plusieurs décors : la cour, la chambre d'Orsino, la chambre d'Olivia, à la manière d'un plateau de théâtre, ce qui a pour effet de naturaliser, voire de banaliser le merveilleux de l'Illyrie mystérieuse et fantastique du texte.

Tandis que la version de 1988, elle, refuse cet artifice et affiche de cor fait sa théâtralité d'une manière beaucoup plus soulignée : un seul ct même espace y représente les différents lieux de l'action, et nous ne voyons pas l'intérieur des deux châteaux. Les deux seuls moments où la caméra ne cadre pas la cour devant le perron sont, d'une part, les arrivées sensationnelles de Viola-Césario (par laquelle le film débute) et de Sébastien, d'une part, cl, d'autre part, la confrontation entre Malvolio enfermé et Feste-Topaz. Donc, même s'il s'agit de théâtre filmé, seule la caméra peut nous montrer un plan serré du visage de Viola en ondine naufragée au cœur chaviré, ou bien l'intérieur de la cave, cadrant le soupirail et le jour qui filtre par les barreaux, ainsi que le profil de Malvolio en plan serré. La caméra souligne par ces plans serrés sur des personnages effondrés la lecture tragique de la pièce qui caractérise cette version.

Le film de 1997 joue davantage sur la convention du film d'action : un très grand nombre de scènes sont tournées en extérieur. Le spectateur est libéré de la distance critique établie par la dénudation de la mise en scène théâtrale et entre de plein pied dans l'invraisemblable et le merveilleux.

\section{L'accessoire}

Les accessoires, quant à eux, ont une fonction attributive au théâtre comme au cinéma. Ils constituent un spectacle à eux tout seuls ${ }^{4}$, et véhiculent une forte valeur de synecdoque qui cristallise des connotations 
sémantiques éparses en figures iconiques stables et aisément repérables.

L'accessoire joue alors le rôle du détail en peinture ${ }^{5}$. C'est là tout l'art de la mise en scène et du choix des accessoires. Trevor Nunn explique l'usage qu'il fait de la perruque pour signifier la vanité de Malvolio ${ }^{6}$, accessoire supplémentaire qui vient s'ajouter aux fameux bas jaunes ornés de jarretières croisées. Dans la production de 1981, Malvolio (Sir Alec Guinness) arbore un chapcau noir à plume, et une lourde chaîne en or tirée tout droit du portrait de Cecil, Lord Burghley, le conseiller de la reine Élisabeth I, dont il joue avec une préciosité toute féminine (code du maniérisme).

Le décor peut s'analyser comme une somme d'accessoires tissant un réseau complexe de détails qui se fondent dans le cadre, et, dans les films cxaminés, on pourrait proposer une typologie en ce qui concerne le choix des boutcilles de vin (l'alcool et les bouffons), celui des costumes (la sexualité, par excmple, et l'androgynic), ou encore le détail s'alliant au geste (Malvolio et sa chaîne en or dans la version de 1981, son chapeau dans la version de 1997). En somme l'accessoire et son équivalent à l'image : le détail constitue la charpente indispensable de l'effet de réel, puisque le cinéma ne saurait être que figuratif, et par conséquent, collection d'objets, autant que d'effets.

Cependant, parmi les trois films étudiés ici, le choix d'un décor d'intéricur ou d'un décor d'extérieur modifie tout à fait le ton de la pièce, et souligne l'importance du paratexte pour le spectateur non seulement eu égard à l'exactitude ou l'incongruité de l'accessoire/détail, mais aussi en ce qui concerne les effets citationnels plus larges.

\section{Le paratexte}

Le film de 1981 est dans la tradition de Hollywood, c'est-à-dire, selon nous, des Préraphaélites, via l'art de DeMille ${ }^{7}$. Il est tourné en intérieur, pour le petit écran, certes, mais aussi pour tirer parti d'un décor reconstitué marqué par le goût des boiseries et du médiévisme gothique d'un mobilier revisité par William Holman Hunt et William Morris. Le nocturne y est symbolisé par l'obscurité des pièces, l'exiguïté des escaliers et des recoins divers où se tiennent les personnages. Une brève comparaison avec le décor de la production de la BBC, plus récente (1989), montre la distinction qui est faite de nos jours entre le moyen-âge et l'époque Tudor, ce qui tend à donner aux costumes une tonalité plus proche de la Renaissance italienne et de la cour des Valois, ainsi qu'un meilleur éclairage rappelant les jeux de lumière en perspective d'un Vermeer. Par comparaison, le film de 1981 est étrangement médiéval, d'un médiévalisme néo-victorien à la fois faussement pédant et réaliste à l'excès. Dans les deux films, 1981 et 1989, toutefois, la nuit est présentće comme un cadre faisant ressortir les effets de lumière d'un clair obscur académique, connotant la nuit des rois d'une manière festive.

Tout à l'opposé de ces espaces intimistes, le film de 1988, nous l'avons 
dit, est tourné sur un platcau de cinéma, mais en extérieur. Nous sommes dans un patio devant une villa italienne, orné de statues classiques, et tapissé d'une neige le plus souvent bleuie par la nuit. Le caractère ouvert de l'espace scénique donne à l'interprétation moderniste des personnages (Feste se donne des allures de pochard aviné rappelant le théâtre de Pinter d'une manière fort peu élisabéthaine) un caractère glacial qui renforce le ton grinçant et l'allure grimaçante des acteurs affichant une théâtralité provocante, voire «persiflante». L'étrangeté du décor connote le conte merveilleux, certes, tandis que l'impression de froid polaire est ironiquement tout le contraire de la chaude intimité du salon, voire du lit, où se trouve le spectateur du petit écran.

Tandis que le film néo-victorien de 1981 fait référence à Hollywood et aux réussites de la mise en scène classique devenues une loi du genre depuis Griffith et Cecil B. DeMille, celui de 1988 (Branagh) fait référence aux théories structuralistes et sémiotiques à la mode dans les années 80 , voire même au modernisme et son avatar, le post-modernisme. La version de 1997, tout au contraire, tournće en extérieurs naturels, fait référence au film de cape et d'épée qui revient en force aujourd'hui (cf. par exemple Le Bossu de P. de Broca, 1998).

Cette étude des trois options choisies pour construire l'illusion d'un réel romanesque montre que tantôt la transposition utilise un décor emprunté au réalisme traditionnel du studio (1981), tantôt elle se coule dans unc métaphore visuelle de la théâtralité comme spectacle en soi (1988), ou encore, clle emprunte au cinéma de cape et d'épée toute sa fougue juvénile (1997).

On pourrait même aller jusqu'à classer les effets de sens du paratexte ên trois catégories : la peinture (1981 et les Préraphaélites; 1988 et Salvador Dali ; 1997 et la peinture classique - on voit une composition à la Velasquez à un moment du film), le théâtre (1981 : classicisme de Stratford : 1988 : modernisme de Pinter ; 1997 : substitution du roman au théâtre), et le cinéma (1981: Hollywood - citations de Cecil B. DeMille, du péplum et du livre illustré ; 1988 : le cinéma britannique du Pop $\operatorname{Art}^{8}$; 1997 : James Ivory et le roman).

L'art de la transposition du texte spectatoriel dépendrait alors d'un paratexte qui lui fournirait en quelque sorte ses lettres de noblesse.

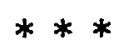

\section{L'image et l'histoire}

La bande-image elle-même est fort différente de la scène de théâtre et impose à la transposition des règles cinématographiques strictes, qu'il s'agisse de la diégèse - examinée ci-dessous - ou du discours, point sur lequel nous reviendrons plus loin. 
Le spectateur de cinéma est doté d'une grande autonomie par rapport à la dićgèse. Il jouit du don d'ubiquité spatio-temporelle en quatre dimensions, pour ainsi dire, grâce à l'image.

\section{L'ubiquité spatiale}

En effet le spectateur de cinéma peut voir les personnages sous des angles fort différents qui n'ont aucun rapport avec l'angle pédestre auquel notre vision théâtrale est soumise. Sans doute, logés au "paradis», nous avons une vue plongeante sur les acteurs : mais qu'en est-il de la contre-plongée? Sans parler du panoramique et du travelling !

Dans la version de 1981 (Sichel), la caméra est remarquablement fixe, sans doute du fait du décor de studio qui interdit de cadrer autre chose que lespace scénique, sous peine de dévoiler le dispositif. Bien plus, le cadrage connote le style Préraphaélite. En effet on est frappé par l'importance accordée au tout premier plan lorsque Orsino flirte avec Césario : les personnages sont très près de la caméra, et occupent tout l'espace du champ visuel, adossés à un arric̀re-plan bloquant le champ à la manière d'un écran. Ce type de profondeur aplatic est caractéristique des scènes néo-médiévales peintes par W. H. Hunt ou Millais (à partir de 1848 et jusqu'à la fin du siècle), scènes tirées de la Bible, mais aussi de Shakespeare, et entièrement reconstituées jusque dans le moindre détail avant d'être recopiées avec une exactitude et une minutie cxtrêmes sur la toilc - témoignant ainsi d'une intuition prémonitoire tout à fait saisissante de ce qu'allait réaliser le cinéma une décennie plus tard. Dans la version de Twelfth Night de 1981, la scène entre Sir Toby, Maria et Sir Andrew est en effet également confinće dans un espace sans profondeur. L'entréc en scène de Malvolio se fait grâce à un escalier, mais le cadrage souligne l'exiguiité «médiévale» du décor Préraphaélite, et la verticalité de la composition cn rappelle le goût pour les écrans supprimant toute perspective, et projetant le personnage (surtout chez Rossetti, certes) sur le seuil du tablcau. Tout ceci donnait aux spectateurs d'alors l'impression qu'ils allaient venir à leur rencontre ${ }^{9}$. Et de fait, la fixité de la caméra dans ce film nous positionne devant un univers pictural plutôt que théâtral, nous privant d'une manic̀re soulignée de toute autonomie, ce qui a pour effet de nous rappeler à quel point cette ubiquité surnaturelle dont nous dote la caméra est également un carcan qui nous emprisonne et nous conditionne.

La légèreté et la fantaisie qui émanent du texte élisabéthain sont totalement absentes de la caméra qui se veut transparente, pur reflet du spectacle ; il faut cependant avouer que la diction des acteurs est en soi un délice et que la rigidité de l'œil-caméra en rehausse la précision et les nuances. Il ne s’agit pas seulement de tableau vivant et de peinture filmée mais de théâtre écouté. Par comparaison, la version de la BBC citée plus haut (de 1989) est beaucoup plus animée et souple, la caméra se partageant également 
entre scènes d'intéricur et scènes d'extérieur de sorte qu'elle devient tout à fait invisible.

Le Twelfth Night de 1988 (Branagh) joue davantage sur l'image de cinéma grâce au changement d'échelle de plan que permet le montage, de sorte que le décor, qui lui, ne change pour ainsi dire jamais, acquiert par ces jeux cinématographiques une grande pluralité de sens. Alternant avec les plans d'ensemble et de demi-ensemble, les plans serrés cadrant le visage des acteurs soulignent les jeux d'expression - visage de Feste chantant, de Malvolio indigné, heureux ou bien humilié, de Viola/Césario en amoureux transi soit par artifice (Viola jouant Césario) soit naturellement (Viola scule) - ct mettent ainsi l'accent sur les sentiments des personnages: surprise, mélancolie, et vanité surtout ${ }^{10}$. En outre le regard à la caméra est utilisé à de nombreuses reprises, surtout pour Feste (11.3) et Césario (11.2; 111.1), c'est-àdire pour orchestrer, par un effet de transgression des «feux de la rampe», les sentiments qu'ils expriment parfois longuement (le texte des inonologues est restitué intégralement).

Un effet sinon de cacophonie, du moins de violente rupture de ton, est ainsi créé, car ces plans serrés vont parfois de pair avec de véritables hurlements chez Feste comme chez Césario. Cela entraîne une violente rupture de ton entre ces cris et le regard à la caméra qui souligne les monologues à peine audibles que susurrent les deux acteurs. Le contraste entre voix tonitruante et voix à peine audible, élocution martelée et diction du bout des lèvres, apporte un contrepoint sonore au jeu du montage cinématographique. Le film acquiert une sorte de violence blanche qui est extrêmement efficace ct dérangeante.

L'adaptation, ou plutôt la transposition de 1997, tire parti de l'ubiquité de la caméra pour nous faire parcourir des lieux réputés inaccessibles: l'entrepont d'un navire (en studio), le fond de la mer, la chambre de Césario) (un inédit dans les représentations de la pièce !), celle d'Olivia ct celle d'Orsino, les salons des deux châteaux, ou encore de folles équipées au bord de la mer se déroulant lors de déplacements savamment orchestrés entre les deux. Nous allons également à l'église et dans un jardin. Tous ces lieux sont tournés en décor naturel : les perspectives changeantes, la variété des décors d'intérieur et d'extéricur nous font voyager presqu'en touristes visitant ce qui semble être une belle demeure anglaise du dix-huitième siècle.

\section{L'ubiquité de l'ail du spectateur et le temps dramatique}

A ce don d'ubiquité spatiale le cinéma ajoute notre émancipation du temps chronologique. Grâce au montage parallèle, par exemple, nous pouvons nous trouver dans deux lieux et deux temps différents, ou au contraire dans deux lieux différents en un même moment. Si les deux versions pour la télévision ne se libèrent que très peu de la chronologie de la pièce (Sichel suit 
toutes les scènes, Branagh en change très peu), celle de Trevor Nunn prend au contraire de grandes libertés avec la chronologie du texte.

En effet, dans la version pour le grand écran de 1997, les quatre premières scènes du deuxième acte sont imbriquées les unes dans les autres avec une complexité d'effets qui trouve sa justification dans une mise en scène "à la Truffaut» de l'émotion amoureuse. Un plan très serré sur Olivia et Césario de profil sert de support au texte («willow cabin speech»); or, simultanćment, pour ainsi dire, nous voyons quelques plans rapides sur Sébasticn et Antonio échangeant quelques paroles (ce qui suggère la passion d'Antonio pour le jeune héros), Toby et Andrew escaladant un mur (passion festive débridée), Malvolio dans un fauteuil confortable lisant une revue intituléc "Amour» à la lumière d'une chandelle, Orsino et Césario chantant accompagnés par Feste («O’Mistress mine»), Olivia au lit rêvant de son amour pour Césario, puis Orsino et Césario jouant aux cartes (plan serré sur Césario amoureux d'Orsino), Toby, Maria et Feste (ubiquité comique du bouffon), et enfin Maria proposant de prendre Malvolio au piège de sa propre vanité au moyen d'une fausse lettre. Le montage alterné très rapide montre chaque membre de la petite communauté se livrant à son occupation favorite la nuit, une nuit festive pour certains (Toby, Andrew et Maria), sentimentale pour d'autres (Orsino et Césario, Malvolio, ou encore Olivia), et scandée par les chansons de Feste qui passe d'un château à l'autre sans que le spectateur y voit d'inconvénient.

Des trois transpositions, celle de 1997 est celle qui exploite le plus librement les possibilités du montage tout comme celles des mouvements de caméra et des plans séquences. La dénudation de la caméra devient une source de plaisir pour le spectateur qui se souvient qu'après tout, le cinéma est aussi l'héritier de Mćliès le prestidigitateur. Elle nous rappelle également que la transposition relève du discours et non seulement de l'image.

\section{Le discours de la transposition: ellipses et scènes}

Le mode temporel de la diégèse théâtrale et filmique s'inscrit ici dans un paradigme que nous appellerons celui du raccourci, comportant d'une part l'ellipse ct, d'autre part, la scène. Il s'agit là d'un problème central à la transposition puisque le texte est souvent découpé, raccourci, ou soumis à des inversions, comme c'est le cas pour le film de 1997 dont il vient d'être question. Le principe en est aristotélicien, bien entendu, à savoir, représenter des idées abstraites par un enchaînement logique (cause-conséquence) du diégétique. Par exemple, avant/après devient porteur d'effets de causalité, ou cncore prolepse/analepse sont investis d'effets de motivation (explications $a$ posteriori, imbroglios, prémonitions, etc.). 


\section{L'ellipse}

Le raccourci par l'ellipse temporelle pose un problème particulicr, puisqu'il implique de la part du spectateur un savoir sur le texte d'origine, savoir qui peut être soit inexistant, soit au contraire très fortement présent. Ceci est lié au texte dramatique, i.e. verbal, et à sa transposition en représentations non-verbales.

Or ce problème est aigu dans Twelfth Night, puisque les jeux de mots constituent une pièce maîtresse du texte. Ceci est bien entendu lié à la réception par un spectateur moderne de jeux sur des mots dont le sens et la référence se sont perdus. Un exemple suffira à évoquer cette question : Trevor Nunn déplore l'obscurité des paroles de Maria «bring your hand to the buttery bar, and let it drink...» (1.3.65) et décide de trancher en supprimant cette réplique. Il est vrai que les autres adaptations qui ont gardé la réplique comportent des effets saugrenus lorsque Maria illustre le sens de son jeu de mots en posant la main d'Andrew Aguecheek sur sa poitrine, de sortc que le geste explique la gaudriole. Or, tout le problème de la réception iconique apparaît clairement dans une telle superposition lourdement tautologique d'un signe discursif et d'un signe iconique.

\section{La scène significative}

Par opposition à l'ellipse, l'adaptation use aussi de la scène significative permettant de réunir plusieurs effets de sens en un laps de temps plus court. Déjà l'adaptation théâtrale de textes historiques fait usage de ce procédé comme en témoignent les comparaisons entre les pièces de Shakespeare et leurs sources (Richard II, par exemple, ou Roméo et Juliette).

Dans le Hamlet de Laurence Olivier, la découverte par Hamlet du crâne de Yorick prend un relief tout particulier dans la mesure où le personnage ne joue plus la comédie. Déjà dans le texte d'origine, la scène a valeur de synecdoque, ramassant en un seul emblème visuel, fort parlant, toute la philosophie et la sagesse qu'enseigne la dure loi universelle de la mort. On observera que cette scène n'est omise dans aucune des transpositions à l'écran, ce qui montre la valeur spectatorielle de la scène par rapport à l'ensemble de l'œuvre.

Dans les versions de Twelfth Night dont il est question ici, l'utilisation d'une scène pour cristalliser les effets de sens de plusieurs épisodes épars dans la pièce est illustrée par la version de 1997 pour le grand écran. Une scène supplémentaire qui n'existe pas dans la pièce a été ajoutée : au tout début du film, nous voyons Sébastien et Viola danser ensemble au cours d'une fête se déroulant à bord d'un bateau, ainsi qu'il a été rappelé précédemment. Viola porte une petite moustache blonde et des vêtements semblables à ceux de Sébastien : le spectateur voit bien qu'il s'agit de deux acteurs et donc de deux personnages différents, mais cette danse souligne leur ressemblance et leur 
amour l'un pour l'autre, partant, leur gémellité émouvante, gémellité qui va semer le désordre en Illyrie. La chute de Viola par-dessus bord lors de la tempête qui secoue bientôt le navire est suivie de la plongée de Sébastien se précipitant à son secours. Nous assistons alors à un ballet aquatique qui relève du plus pur cinéma, au cours duquel les deux corps se perdent et se retrouvent avant d'être arrachés l'un à l'autre par une mer démontée.

La métaphore visuelle du couple ontologique et de sa perfection platonicienne projette sur tout le film une symbolique de l'arrachement et de la fusion qui atteint son paroxysme avec la réunion des jumeaux au cours de la dernière scène. La fluidité du montage associée à un jeu d'acteurs d'une grande souplesse évoque, dans cette scène de retrouvailles, la grâce émouvante des corps dans la séquence sous-marine du début. Nous finissons par être émus de cette réunion amoureuse au point d'y voir une beauté féerique sans doute très proche du texte élisabéthain tel qu'il est compris aujourd'hui.

Les couples étranges formés par les voies mystérieuses d'un hasard providenticl prennent un sens fécondé par ce couple ontologique à la fois androgyne ct angélique. Rien de tout cela n'eût été possible sans la séquence d'ouverture.

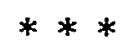

\section{Le corps de l'acteur}

Le corps est lui aussi spectacle et offre de multiples registres de Iransposition du théâtre au cinéma, ainsi que nous allons le rappeler bric̀vement ici. Au théâtre, le corps de l'acteur est cet éternel présent de l'acte de chair que partagent acteurs et spectateurs ; mais au cinéma le corps est ce texte, ce lieu imaginaire où s'investissent tous nos fantasmes les plus inavoućs, les plus nocturnes.

Nous étudicrons d'abord le corps de l'acteur du point de vue des effets de réel qui en découlent au théâtre comme au cinéma. Dans Twelfth Night, le déguisement de l'acteur (un garçon chez les Élisabéthains) en fille puis de cette fille en garçon est source de constante remise en cause du contrat énonciatif spectaculaire. Non seulement le spectateur est constamment invité à anticiper une découverte accidentelle du sexe de Césario, ce qui donne du sel à toutes les situations de dialogue où Césario est directement ou indirectement impliqué, mais la bisexualité du personnage est source d'imbroglios pour le personnage lui-même qui exprime plusieurs fois son désarroi (par exemple lorsqu'il/clle doit se battre en duel ou, plus grave, être aimé). La diégèse haigne dans l'ambivalence.

D'un point de vue plus esthétique, cette bisexualité multiplie également les difficultés de l'acteur qui doit à la fois être féminin en Viola (I.1 par excmple), et être féminin-mais-masculin dans les autres scènes, puis 
masculin-mais-féminin dans la dernière scène où il s'agit d'une femme vêtue en homme.

Une telle duplication n'est pas faite pour être invisible (théorie de l'art classique selon laquelle les marques d'énonciation doivent être effacées) mais au contraire bien visible (théorie de l'art maniériste et baroque selon laquelle les marques d'énonciation sont elles-mêmes des signes à l'adresse du spectateur). La duplication et la bisexualité sont donc un spectacle à part entière et ceci est rendu lorsque c'est le même acteur qui joue les jumcaux, choix de la version filmée de 1981.

Le problème peut toutefois être envisagé d'une manière différente si le spectateur doit seulement trouver du plaisir à l'identification secondaire avec les personnages et non à une identification primaire à la caméra, choix revendiqué par Trevor Nunn ${ }^{11}$. Le spectateur ne sait pas toujours s'il y a effet spécial ou pas ${ }^{12}$, et s'il le sait, cela conforte le contrat énonciatif initial, en le ramenant à la surface de la réception; par ailleurs, si l'effet symbolique est l'universalité de l'amour, la bisexualité et le travesti sont là pour la représenter tout au long du texte, et n'est pas contredit par l'affleurement d'un rappel du cinématographique dans le filmique.

Tel est bien l'effet produit par la version de 1981, où nous voyons la même actrice jouer Sébastien et Viola avec une légère inflexion du timbre de la voix, ce qui a pour effet de rendre présent le corps de l'acteur dans le texteimage et injecte un effet de réel renvoyant à la représentation filmée beaucoup plus convaincant que le jeu par deux acteurs différents. Tel est en effet le choix des versions de 1988, 1989 et de 1997, ce qui a pour effet de déréaliser non pas le jeu de l'acteur mais, plus grave, la crédibilité de l'erreur visuclle et de la problématique de l'ambivalence des apparences.

Discerner un trucage est de l'ordre du spectatoriel (cinématographique dans ce cas) et ne détruit pas le pacte spectatoriel (ou contrat énonciatif), tandis que voir une différence évidente là où les personnages déclarent ne pas en voir ne renforce pas nécessairement notre croyance à la problématique des apparences, ne faisant, somme toute, que nous renforcer dans notre compréhension de l'aveuglement des personnages, aveuglement qui est bien mieux souligné lorsqu'il est motivé diégétiquement par une ressemblance effective dont nous nous portons nous-mêmes garants, paradoxalement, puisque nous voyons que c'est le même acteur.

Le cinématographique vient renforcer le filmique et non le détruire. L'étude de la forte symbolique de la partition et de la réunion des corps dans la version de 1997 l'a d'ailleurs amplement démontré. 


\section{Le discours spectaculaire au cinéma}

Cette émancipation de l'espace et du temps que la caméra permet au spectatcur, et qui entraîne une grande variété de modes de transposition possibles, ainsi que nous venons de le voir, implique en même temps que le spectatcur soit en réalité prisonnier de l'image, c'est-à-dire du plan en deux dimensions que constitue l'écran ${ }^{13}$. L'image jouit d'une dynamique et d'une rhétorique qui lui sont propres. Ce sont les trois axes de la composition fondéc sur le cadre : la verticalité, l'horizontalité et la diagonale.

Sclon nous, le présent dramatique spécifique au théâtre que partagent actcurs et spectateurs, ce troisième temps, est, au cinéma, transposé en un espace-temps dramatique tout aussi unique et spécifique, que partagent spectatcurs (voycurs) et acteurs (fixés par la pellicule photosensible), l'espace bi-dimensionnel de l'écran, lieu privilégié de la représentation des actants de la communication.

Par exemple, dans le Macbeth d'Orson Welles (1948), le chaos provoqué par l'assassinat de Duncan est représenté par une plongée sur la cour du château ct des personnages courant droit devant eux : ils surgissent en haut de l'écran à gauche et disparaissent en bas à droite. La rhétorique selon laquelle le haut est céleste et le bas infernal suggère que ce chaos entraîne une chute vertigineuse.

\section{Un exemple de discours cinématographique : la scène de la lettre}

Dans Twelfth Night, le cadrage de la scène de la lettre est très différent et montre des solutions au problème de l'adaptation qui relèvent justement de cet espace-temps spécifique à l'écran ${ }^{14}$.

Dans la version de Twelfth Night de 1997, un cadrage très serré sur Malvolio et une Vénus de marbre dans un jardin de la Renaissance (haies de huis taillées en labyrinthe) créc un effet irrésistible : la statue est cadrée à la laille, puis plus bas (de la pointe du pubis aux cuisses), et enfin jusqu'aux épaules : elle a les bras coupés. Ces fragments du corps féminin - présentés à la manière de blasons d'amour - ponctuent le contenu de la lettre que lit Malvolio d'un commentaire ironique appuyé, certes, mais volage et drôle.

Dans la version de 1981, cette scène est interprétée, nous l'avons dit, par Alec Guinness qui marie raideur pompeuse et fatuité narcissique du geste comme de la voix avec la subtilité qu'on lui connaît. Le comique vient du jeu d'actcur ct non du cadrage - c'est le code du théâtre filmé, code auquel, nous l'avons dit, la fixité de la caméra ne saurait nous laisser échapper.

Dans la version de 1988 (de Branagh), le jeu des spectateurs intradiégétiques, Toby, Andrew et Maria, est très souligné, et leur amusement frénétique nuit aux effets plus subtils du jeu de l'acteur Richard Briers dont le génic plus tragique que comique s'accommode mal de telles clowneries. 
Le montage parallèle entre Malvolio et les farceurs qui apparaît chez. Sichel et Nunn cède la place à un cadrage des deux partis en présence de chaque côté d'une haie, avec seulement quelques plans serrés sur chacun d'cux par instant. Nous sommes confrontés à un parallèle violent entre la victime stupidement vaniteuse et naïve et les tortionnaires égrillards, ce qui conforte l'impression d'agressivité donnée par les autres scènes. Alors que, curieusement, lorsque dans les deux autres versions, nous voyons les deux partis en plans alternés, ils nous paraissent également drôles, l'un par sa gestuelle efféminée et sa pédanterie (Alec Guinness surtout), et les autres par les expressions hilares et clownesques de leur faciès de pitres.

Nous ne serions pas loin ici de démontrer notre asservissement aux lois de l'effet Koulechov, si ce n'était l'égale rapidité du montage qui occulte cet esclavage de l'ceil.

\section{Le discours cinématographique et le pacte de fiction}

Le spectateur est en effet prisonnier de l'objectif, c'est-à-dire de la monstration cinématographique. Il voit le mouvement et la mise en scène en trois dimensions, certes, mais uniquement par rapport à la caméra qui lui dicte sa position. La profondeur de champ, par exemple, lui est imposée par l'objectif (le Macbeth d'Orson Welles et l'utilisation d'un «selective focus»" 15 est exemplaire à cet égard, tout comme le flou accompagnant l'entréc en scène de Viola dans la version de 1981 de $T N$, ainsi que nous l'avons dit au début de cette étude). D'une manière plus générale, le contrat énonciatif est frappé au sceau des métaphores visuelles où nous plongeons la tête la premièrc.

C'est ainsi que dans le Macbeth de Welles, une forte plongée sur la mixture immonde qui bouillonne dans le chaudron des sorcières oblige le spectateur à quitter le monde ordinaire bien avant même que le film ne commence. Lorsque Macbeth entre dans le champ de la caméra, nous savons que ce n'est plus son entrée en scène qui importe, mais davantage le fait que nous avons nous-mêmes été immergés dans l'univers du film.

Il n'est pas sans intérêt de noter que le film de 1997 commence lui aussi par une plongée sous-marine en apnée, métaphore filée de notre entréc en cinéma. Déjà dans la pièce élisabéthaine, la mer a transformé l'entréc dans le monde de la fiction non seulement des personnages mais aussi des spectateurs, procédé repris dans La Tempête. La métamorphose du spectateur élisabéthain est donc elle-même transposée à l'écran. Les trois films étudiés portent les stigmates de notre métamorphose, mais selon des modes différents.

Notre entrée en «Illyrie», pays des noces perpétuelles de la nuit et de la fête, se fait sur le mode théâtral dans la version de 1981. Mais cela ne saurait s'effectuer sans une manipulation de notre regard, de notre manière de voir : les acteurs entrant devant/dans une scène de théâtre qui se métamorphose sous nos yeux, ainsi que nous l'avons dit. Notre arrivée en Illyrie dans la version 
de 1988 se fait sur le mode pathétique : Viola, visage tuméfié et poitrine dénudée, vêtue de haillons, tourne vers la caméra un visage ruisselant d'eau et de larmes ; elle renifle (!) et s'essuie d'un revers de la main.

Dans le premier cas, le spectateur est au théâtre, et dans le second, le spectateur se croit convié à un film d'aventures sinon hugolesques, du moins dickensiennes. Par contre, le motif de l'immersion comme entrée dans le monde fécrique de la fiction romanesque rappelle d'autres immersions dans les caux noires de la nuit «écranique» (le néologisme est de Metz) : immersion du Macbeth d'Orson Welles, mais surtout du film noir des années 40, parodiée dans Sunset Boulevard.

Lc discours cinématographique constitue, on le voit, à lui seul un domaine où la transposition s'exerce tout à fait librement.

Il est certain que l'interprétation de cette scène d'ouverture (Acte I, scène 2 dans la pièce) modifie considérablement la réception de l'ensemble de la pièce. Autrement dit, dès lors qu'un fragment du texte est transposé et inséré dans le tissu filmique c'est tout le film qui s'en trouve altéré. La trame syntagmatique filmique constitue un discours autonome qui se suffit à luimême et se détache à la manière de tout hyper-texte s'alimentant d'un hypotexte: le sens ne vient plus de l'effet citationnel mais davantage de la prolepse et de l'analepse, c'est-à-dire des références syntagmatiques de chaque fragment du film au monde du film lui-même.

Dans les trois exemples de la lettre que nous avons cités plus haut, cet intertexte devient un contexte pour les autres segments du film. Le comique suggéré par le cadrage dans la version de 1997 demande une participation active du spectateur: nous anticipons les effets puisque nous reconnaissons la statuc dès le début, toutefois nous ne pouvons l'identifier complètement qu'à la fin du travelling: les bras coupés de la Vénus de Milo sont une surprise. Dans la version de 1981, le comique de l'acteur crée un spectacle dans le spectacle: nous nous amusons à la fois du rôle ridicule que se donne Malvolio en lisant la lettre, et de l'interprétation à la fois drôle et curicusement impénétrable qu'en donne la star - Alec Guinness est si connu que le spectateur réagit ici comme devant un Charlot : il rit par anticipation autant que par réaction devant la performance. Dans le film de 1988, le contraste saugrenu entre la haute figure d'un Malvolio au visage sévère dont le sourire contrefait laisse voir d'énormes dents inquiétantes, et les farceurs s'amusant davantage de leur propre farce, et donc de ce qu'ils prennent pour leur propre talent, que du spectacle que leur donne Malvolio, ce contraste connote les multiples miroirs et les fausses perspectives d'un maniérisme outrancier plus angoissant que comique.

Faut-il aller jusqu'à dire que le spectateur est prisonnier du discours cinématographique de la bande image ? Il voit une continuité fictive qui est indépendante du temps de la représentation : cette continuité est celle du 
montage de la bande image et de la bande son, c'est-à-dire de l'espace-temps cinématographique.

\section{Le discours filmique}

Nous nous interrogerons également au sujet du dispositif spectaculaire comme discours filmique et cela à propos d'un cas particulier, celui de la pièce dans la pièce.

Le dispositif énonciatif comique dans Twelfth Night est bien entendu lié au corps de l'acteur, ainsi qu'il a été dit plus haut. Cependant, les jeux sur le verbal et le non-verbal contribuent également à créer des effets comiques, soit par des effets de duplication, soit par une distanciation ironique. Par excmple, dans la pièce, le spectateur apprécie la mise en abyme du théâtre dans le théâtre avec l'entréc en scène de Malvolio déguisé en amourcux aux bas jaunes et noirs, arborant le masque de l'amoureux transi (sourire sempiternel).

Nous avons vu Maria dans le rôle de dramaturge (elle imagine la scène) et dans celui du metteur en scène (didascalies dans la lettre au futur acteur). Nous voyons en outre un deuxième spectacle puisque Maria et ses acolytes épient sur scène les faits et gestes de Malvolio lorsqu'il trouve la lettre et lorsqu'il joue son rôle. De plus, lors de cette performance, un troisième spectacle, celui du spectateur ahuri, dévolu à Olivia, vient s'ajouter aux deux autres.

La duplication des actants de l'énonciation théâtrale est en soi un spectacle, ce qui constitue donc un quatrième plan de représentation dans lequel le théâtre se représente. L'effet est purement énonciatif ct fait référence au théâtre par des procédés non-verbaux reposant sur la différence entre l'espace scénique et l'espace spectatoriel. Ces facéties sur le seuil du manleau d'Arlequin 16 remettent en cause le pacte spectatoriel pour lui conférer une valeur symbolique, puisqu'il est alors question d'illusion d'illusions (vanitas vanitatis).

A l'écran, l'espace est subdivisé en micro- et macro-scènes, les blagueurs s'amusant de l'aveuglement de leur victime : cet effet de sens est rendu par une théâtralité que souligne un mimétisme kinésique (gestuelle, mimiques) et proxémique (distance variable entre les protagonistes : les déplacements de Malvolio entraînent une chorégraphie comique), dans les deux productions télévisuelles.

Mais le montage permet en outre des effets de champs/contrechamps soulignant des plans de réactions auxquels le spectateur s'identifie tour à tour (identification secondaire), partageant la joie des farceurs et l'émoi de l'amoureux transi. Le montage crée de ce fait une dimension spectatoriclle spécifique au cinéma, puisqu'il y a une focalisation plurielle dans ce cas ${ }^{17}$. Le spectateur du film est donc à même d'occuper les deux rôles, celui de l'amusé et celui du médusé, ce qui au théâtre n'est pas envisageable, la victime y 
constituant un spectacle occupant un espace scénique à part entière.

$* * *$

Ces quelques exemples suffisent à montrer l'étendue de la problématique de la transposition, les codes filmiques véhiculant leurs propres signifiés avec unc complexité accrue au fil des années, générant un paratexte sans cesse renouvelé, complexifiant l'hypo-texte en de multiples ramifications, comme la comparaison de trois films de 19811988 et 1997 l'a prouvé.

C'cst cependant surtout sur la fonction symbolique du spectatoriel théâtral ct cinématographique/filmique que repose le sens de ces textes. Autreinent dit, l'cuvre est non seulement ouverte, elle est aussi fermée, et nous impose un ordre spécifique, un monde à la fois possible et original. Nous l'avons vu plus haut, le film de 1997 réussit à transformer la pièce en un poème d'amour et de réconciliation. On peut toutefois se demander si la pièce n’est pas davantage axée sur la nuit que cette version ne le laisse paraître.

En effet, l'effet spectaculaire de la pièce élisabéthaine véhicule un état instable où les erreurs sont constamment mises en avant et que symbolise en partic la métaphore visuelle de la nuit.

Olivia épouse Sébastien en croyant épouser Césario, Sir Toby et Sir Andrew s’en prennent à Césario alors qu'ils ont été corrigés par Sébastien, ou encore Césario/Viola risque d'être puni/ie cruellement par Orsino alors que, dans un brusque revirement, ce dernier finit par mettre son destin entre ses mains. L'erreur qui s'empare de l'Illyrie avec l'arrivée de Viola se répand comme une contagion que le personnage sème autour de lui. Cette erreur prend les apparences d'un incendie amoureux qui les enflamme sans raison.

La version de la BBC (1989), à laquelle il a été fait rapidement allusion, rend hien celte instabilité des signes, en particulier l'instabilité de l'humeur de chacun, par de nombreux plans serrés sur les personnages et un jeu d'expressions changeantes sur leurs visages. Seul le visage de Malvolio (Alec MacGowen) se crispe de plus en plus, alors que celui de Césario (Felicity Kendal) fait montre d'une remarquable mobilité d'expression. Or cette transposition de l'humeur instable et irrationnelle, investie dans les jeux de mots du texte, sur la page vide des visages est typiquement filmique, le visage connotant la surface de l'écran et les contaminations de sens qui s'y produisent du fait du montage, mais aussi l'écran imaginaire et le spectacle de l'inconscient, du désir, bref, des passions. De ce point de vue, la production de la $\mathrm{BBC}$ est beaucoup plus expressive et spectaculaire au sens propre que tous les artifices de la production de Branagh peuvent espérer l'être.

Si les passions sont en effet spectaculaires dans Twelfth Night, ainsi que le signifient les brusques revirements, les erreurs et les aveuglements 
multiples, le cinéma possède un registre de représentation unique dans cc domaine avec le plan serré, voire le plan détaillé sur un regard ou une moue qui véhiculent l'expression des sentiments, ainsi qu'il a été suggéré plus haut ${ }^{18}$. L'image «coup de poing»d'un visage rendu «expressif» n'a pas son pareil, surtout lorsqu'il est accompagné d'un regard à la caméra.

Mais comme nous l'avons vu, le montage permet de rendre cette folie collective et nocturne avec une grande variété d'effets selon les options choisies. Dans le film de 1997, le montage parallèle très serré créc une impression de fête nocturne déclinée sous toutes ses formes, dans celui de 1988, le nocturne rayonne d'une blancheur létale et glaciale qui se retrouve sur les crispations affectées du visage de marbre d'Olivia, la mélancolic hirsute d'Orsino, et surtout la rage froide de Feste. Seule Viola verse de chaudes larmes sur la disparition puis sur le retour de Sébastien. Dans la version de 1981, la nuit est réaliste: c'est l'intimité d'un coin de table, les rires des amis d'enfance, la tendresse d'Orsino et d'Olivia pour Césario/Viola el la faconde bon enfant d'un Feste toujours souriant.

Toujours est-il qu'au cinéma la nuit à l'écran bénéficie d'un allić sûr. l'obscurité de la salle. En fin de comptc, au cinéma, le spectateur n'en finit pas de célébrer les noces de la nuit et de la fête. Comme l'écrivait Jacques Spitz, le cinéma «vient à moi. Fait d'images, c'est-à-dire de la matière même des songes, il s'offre comme le combustible idéal du foyer intérieur" ${ }^{19}$. Sans doute était-ce déjà là ce que Twelfth Night signifiait pour les Élisabćthains.

Raphaëlle COSTA DE BEAUREGARD Université Toulouse-le-Mirail

\section{N O T E S}

${ }^{1}$ John Sichel, Twelfth Night: Sir Alec Guinness (Malvolio), Sir Ralph Richardson (Sir Toby Belch), Joan Plowright (Viola), Adrienne Corrie (Olivia), Gary Raimond (Orsino), Tommy Steele (Feste), John Moffat (Sir Andrew Aiguecheek), ITC Entertainment, 103 min. 1981. Video Polygram 1992 (il existe une version de la BBC de John Gorrie de 1989 que nous n'analysons pas ici à défaut de place, quoiqu'elle mérite une étude par la qualité de son thêâtre à la fois classique et très nuancé). Kenneth Branagh, Twelfth Night avec Richard Briers (Malvolio), Caroline Langrishe (Olivia), Frances Barber (Viola), Christopher Ravenscroft (Orsino), James Saxon (Sir Toby Belch), Anton Lesser (Feste), James 
Simmons (Sir Andrew Aiguecheek) pour la Renaissance Theatre Company Thames Television. 156 min.. (1988). Video Thames Television International/Video Collection International, 1991. Trevor Nunn, Twelfth Night avec Helena Bonham Carter (Olivia), Richard E. Grant (Sir Andrew Aguecheek), Nigel Hawthorne (Malvolio), Ben Kingsley (Feste), Mel Smith (Sir Toby Belch), Imogen Stubbs (Viola), Steven Mackintosh (Sebastian). Renaissance Film Production. $128 \mathrm{~min}$. 1997. L'ordre d'apparition des acteurs dans les listes ci-dessus veut refléter leur célébrité au moment de la sortie du film. Pour l'approche méthodologique utilisée dans cet article, voir André Helbo, L'adaptation - Du théâtre au cinéma, Paris, A. Colin, 1997. p. 9-13. Cet ouvrage reprend avec clarté les différents débats et fait le point d'une manière très utile. Voir aussi Christine Hamon-Sirejols, Jacques Gerstenkorn et André Gardies, Cinéma et théâtralité - Cahiers du GRITEC, Lyon, Aléas, 1994.

${ }^{2}$ Ce néologisme est dû à E. Souriau. Voir D. Chateau, «Film et réalité : pour rajeunir un vieux problème», IRIS, vol.1, n⿳1, 1983, p. $51-66$ et D. Sipière, «Où est la réalité des objets?», éd. R. Costa de Beauregard, Le Film et ses objets()bjects in Film - La Licorne, Poitiers, UFR de Langues et Littérature, 1997, p. $33.35-348$.

${ }^{3}$ Roland Barthes, La Chambre Claire - Note sur la photographie, Cahiers du Cinćma, Paris, Gallimard - Seuil, 1980. Sur la trace photographique et le cinéma, voir J.L. Leutrat, Vie des fantômes - Le fantastique au cinéma, Paris, Cahiers du cinéma, 1995 et notre «Traces de Stars: compétences spectatorielles et spéculaires dans Opening Night de John Cassavetes (1978)», éd. R. Costa de Bcauregard, Le Cinéma se Regarde: Spectacle et Spécularité, Toulouse, Iniversité Toulouse Le Mirail, 1995, p. 63-73.

4 Jacques Gerstenkorn, «DeMille et le spectaculaire: une esthétique du détail», éd. C. Hamon-Sirejols et als., Le Spectaculaire, Cahiers du GRITEC, Lyon, Aléas, 1997, p. 145-54.

${ }^{5}$ D. Arasse, Le Détail - Pour une histoire rapprochée de la peinture, Paris, Garnier-Flammarion, 1996 (1992).

"François Laroque, "Vérités et mensonges - Entretien avec Trevor Nunn», éd. Roger-François Gauthier, Shakespeare: La scène et ses miroirs - Théâtre aujourd'hui, n6, Paris, CNDP, 1998, p. 226-30.

7 Voir notre «La problématique du figuratif au cinéma. Étude de cas : Le Moïsc de Cecil B. DeMille et l'art préraphaélite», éd. Jean-Marie Marconot, Le héros e't l'héroïne bibliques dans la culture, Montpellier, Université Paul-Valéry, Centre de Recherche Biblique Interdisciplinaire, 1997, p. 195-209.

${ }^{8}$ E. de Cacqueray, Individu et société dans le cinéma britannique des années soixante aux années quatre-vingt : l'image-texte, Thèse, Toulouse-le-Mirail, 1997

${ }^{9}$ Jeremy Maas, William Holman Hunt and The Light of The World (1984) Aldershot, Wildwood House \& Cambridge, Cambridge University Press, 1987. p. 232 .

${ }^{10}$ Gilles Menegaldo (éd.), L'expression du sentiment à l'écran - La Licorne, Poitiers, UFR de Langues, 1995. 
11 «I was told the great advantage of cinema is that you can use one person to play both roles, and you can do it with technology. I knew I didn't want that, for two reasons. [...] an audience always knows when technology is involved, always withholds a little bit of their belief and their investment in the characters because [...] there's trickery. [...] Shakespeare's subject is immediately clear : what's the difference between a girl loving a man and a boy loving a man ...", "Interview given by Trevor Nunn, Direcior of the Film Twelfth Night to François Laroque".

12 Pour une typologie des effets de sens du trucage, voir Christian Metz, Essais sur la signification au cinéma, 2 vols, Paris, Méridiens Klincksieck, 1972, vol. 2 , p. 173-92.

13. «Un des apports les plus intéressants à la théâtrologie contemporaine a été fourni par la mise au point du concept de texte spectaculaire. Si l'on entend par là toute combinatoire régie par des règles de fonctionnement (sémiotique) réciproque (les structures relevant du parcours sémantique inscrit dans le texte), on tient dans cet opérateur un instrument qui permet d'aborder la performance théâtrale sous l'angle matérialiste de l'événement en actes. On sait aussi que ce paramètre constitue un trait distinctif de la représentation théâtrale. Le film privilégic la fiction, ignore la dimension de performance inprasentia, renvoic à un scénario dont la fonction ne recouvre pas totalement celle du texte théâtral», Helbo, L'adaptation, op. cit., p. 103.

14 Pour une définition de cet écran métaphore visuelle de l'espace-temps spécifique au cinéma, voir Christian Metz,Le Signifiant imaginaire, Paris, Christian Bourgois, 1993 (1977) et L'énonciation impersonnelle ou le sue du film, Paris, Méridiens-Klincksieck, 1991.

is Il s'agit du type de profondeur de champ utilisé avant le grand-angle qui allait s'imposer dans les années 50 avec Citizen Kane (1941): «Selective focus makes the faces sharp and the foreground and the background planes hazy. $|\ldots|$ Selective focus typically draws the viewer's attention to the main character or object». Le grand-angle, «deep focus», donne au contraire une profondeur de champ égale du premier au dernier plan de l'image. Il est intéresant de noter que le Macbeth de Welles est de 1947, mais que la profondeur de champ utilisée est connotée «traditionnelle» par rapport au grand angle de Citizen Kane. Le choix esthétique n'est pas innocent, puisqu'il s'agit de représenter à la fois le monde de l'hypocrisie où le personnage est plongé et sa vision personnelle marquéc par le cauchemar et l'obsession, ambivalence de la vision que le flou au premier plan et celui à l'arrière-plan soulignent continuellement dans le film.

${ }^{16}$ Helbo, L'adaptation, op. cit., p. 45-9.

${ }^{17}$ François Jost, L'ril-caméra, Lyon, P.U.L., 1987 ; l'auteur distinguerait ici entre focalisation et ocularisation, nous renvoyons donc le lecteur à cet ouvrage très éclairant.

${ }^{18}$ Voir à ce sujet C. Murcia et G. Menegaldo (éds.), L'expression $d u$ sentiment au cinéma, La Licorne, Poitiers, Université de Poitiers, 1996, et J. Aumont, Du visage au cinéma, Paris, Éd. de l'Étoile/Cahiers du cinéma, 1992.

19 Jacques Spitz, «Chronique de Londres», Revue du Cinéma, n¹3, le série - 2e année - Jer août 1930, p. 72-7. 